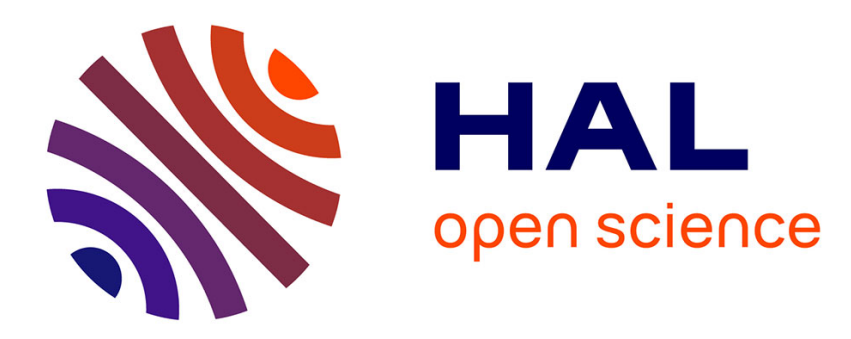

\title{
THE OMEGA PHASE TRANSFORMATION
}

J. Sanchez, D. de Fontaine

\section{To cite this version:}

J. Sanchez, D. de Fontaine. THE OMEGA PHASE TRANSFORMATION. Journal de Physique Colloques, 1977, 38 (C7), pp.C7-444-C7-452. 10.1051/jphyscol:1977788 . jpa-00217292

\section{HAL Id: jpa-00217292 https://hal.science/jpa-00217292}

Submitted on 1 Jan 1977

HAL is a multi-disciplinary open access archive for the deposit and dissemination of scientific research documents, whether they are published or not. The documents may come from teaching and research institutions in France or abroad, or from public or private research centers.
L'archive ouverte pluridisciplinaire HAL, est destinée au dépôt et à la diffusion de documents scientifiques de niveau recherche, publiés ou non, émanant des établissements d'enseignement et de recherche français ou étrangers, des laboratoires publics ou privés. 


\title{
THE OMEGA PHASE TRANSFORMATION
}

\author{
J. M. SANCHEZ and D. DE FONTAINE
}

School of Engineering and Applied Science, University of California, Los Angeles, CA. 90024, U.S.A.

\begin{abstract}
Résumé. - On examine divers modèles théoriques pour la transformation $\beta \rightarrow \omega$ dans des alliages à base de $\mathrm{Ti}, \mathrm{Zr}$ et $\mathrm{Hf}$, et l'on compare les prévisions théoriques avec les résultats expérimentaux. Quoique les causes fondamentales de cette transformation ne sont pas bien connues à présent, la plupart de ses caractéristiques peuvent être décrites de façon assez précise au moyen de modèles phénoménologiques. On présente, entr'autres, un modèle récent basé sur la méthode Cluster Variation où l'on se sert de la cellule primitive c.c. comme motif atomique de base. Les calculs montrent que la concentration de complexes activés, responsables de la diffusion, augmente lorsque l'on s'approche de la température de transformation $\beta \rightarrow \omega$. Ceci explique l'augmentation de la vitesse de selfdiffusion ob̆servée à basse température dans les métaux Tí, Zr et Hf. En outre, ce modèle, basé sur l'existence de fluctuations de type $\omega$ dans la phase $\beta$, tient compte quantitativement des anomalies de diffusion observées dans les phases c.c. $(\beta)$ des métaux de transition du groupe IV B.
\end{abstract}

\begin{abstract}
Different theoretical models for the $\beta \rightarrow \omega$ phase transformation in $\mathrm{Ti}, \mathrm{Zr}$ and $\mathrm{Hf}$ alloys are reexamined and their predictions compared to experimental evidence. Although the fundamental reasons for the presence of the transformation are not fully understood at the present time, most of the features of the transition can be described quite accurately by phenomenological models. A brief discussion is presented of a recent Cluster Variation calculation which uses the bcc primitive unit cell as the basic configurational cluster. Such a calculation shows an increase in the concentration of activated complexes for diffusion as the $\beta \rightarrow \omega$ transition is approached, thus explaining the enhancement in self-diffusion at low temperatures observed in $\mathrm{Ti}, \mathrm{Zr}$ and $\mathrm{Hf}$. The diffusion model based on the presence of $\omega$-like fluctuations in the $\beta$-phase is, in addition, in excellent quantitative agreement with rather puzzling observations in $\beta$-stabilized alloys of the Group IV B transition metals.
\end{abstract}

1. Introduction. - The group IV B transition metals $\mathrm{Ti}, \mathrm{Zr}$ and $\mathrm{Hf}$, when alloyed with a bec $(\beta)$ stabilizer element (e.g., $\mathrm{Nb}, \mathrm{V}, \mathrm{Fe}, \mathrm{Mo}, \mathrm{Mn}, \mathrm{Cr})[1,2]$, or pure $\mathrm{Ti}$ and $\mathrm{Zr}$ at high pressures $[3,4]$ can transform at low temperatures to a metastable hexagonal phase, the so-called $\omega$ phase. Since the early work by Frost et al. $[5,6]$ on $\mathrm{Ti}-\mathrm{Mn}$ and $\mathrm{Ti}-\mathrm{Cr}$ alloys, it became clear that the $\omega$ phase was one of the most conspicuous features of the Group IV B transition metals.

A number of theoretical models have been proposed to explain the broad range of phenomena associated with the $\omega$ phase [7-12]. At the time of writing, however, a theory of the $\beta \rightarrow \omega$ phase transformation based on first-principles is still lacking. Thus, the theoretical contributions have been in the form of phenomenological models which, notwithstanding, describe quite accurately most of the features of the transition.

Although the crystallographic relationship between the bcc and $\omega$ phase is particularly simple, much experimental work was required in order to elucidate the omega structure [13-16]. The reason for early controversial reports on the structure of the omega phase is that the metastable phase can be formally obtained from the bce lattice by means of a simple longitudinal displacive wave of magnitude $a / 6$ ( $a$ is the bcc lattice parameter) and wave vector

$$
\mathbf{k}_{\omega}=2 / 3 \text { [111] [17] . }
$$

Thus four variants of the omega phase exist, one for each $\langle 111\rangle$ direction, and one must be careful in assigning the observed reciprocal lattice reflection to the proper variant.

The alternate collapse of pairs of (111) planes produced by a $\mathbf{k}_{\omega}$ displacive wave with an amplitude slightly smaller than $a / 6$ is schematically shown in figure 1. Complete plane collapse, which corresponds to the omega phase, results in a two-layered structure of the $\mathrm{AlB}_{2}$ type. A change in sign in the $\mathbf{k}_{\omega}$ displacive wave (broken line in figure 1) gives rise to a different (high energy) structure, termed antiomega $(\omega)$ by Cook [9-11]. Within a given variant, three different subvariants or domains may develop, since three distinct types of plane collapse can occur : $(\alpha, \beta)$, $(\beta, \gamma)$, or $(\gamma, \alpha)$, each giving rise to a particular omega subvariant, $\alpha, \beta, \gamma$ denoting the stacking sequence of $\langle 111\rangle$ planes in the bec lattice.

The nature of the $\beta \rightarrow \omega$ phase transformation has been revealed by electron diffraction in a large number 


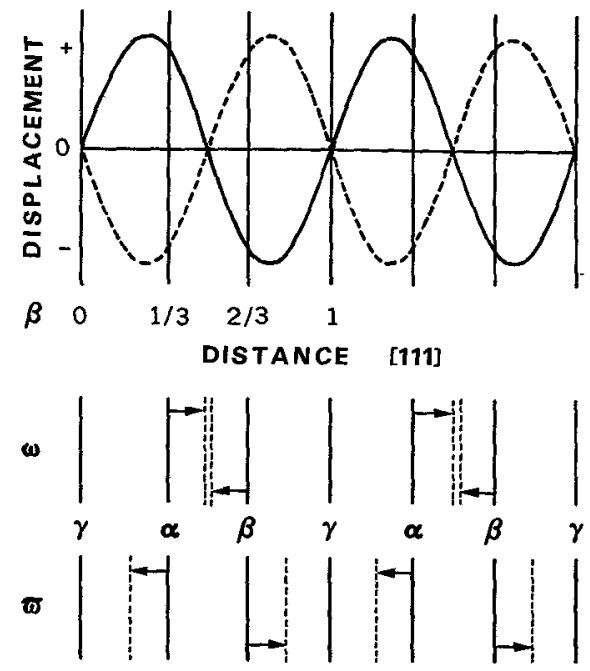

FIG. 1. - The effect of the $2 / 3[111]$ displacive wave on the boc lattice.

of $\mathrm{Ti}, \mathrm{Zr}$ and $\mathrm{Hf}$ base alloys [18-21], and by neutron [22] and X-Ray diffraction and Mössbauer [23] spectroscopy in the $\mathrm{Zr}-\mathrm{Nb}$ system at different concentrations and temperatures.

Early electron diffraction experiments showed that considerable diffuse intensity was present near the omega superlattice reflections in a concentrationtemperature range where the bcc structure was known to be the stable phase [18]. On a $(110)_{\beta}$ reciprocal lattice section, the diffuse intensity appeared as streaks perpendicular to the $\langle 111\rangle$ directions and slightly offset from the omega superlattice reflection towards the octahedral [111] reciprocal lattice points. In some $\mathrm{Ti}$ and $\mathrm{Hf}$ alloys, the offset and elongation of the diffuse intensity was compounded with strong curvature, so that the streaking resembled a sphere of intensity centered at the octahedral positions of the bcc reciprocal lattice of $\beta$, with a radius slightly smaller than $g / 3, g$ being the length of the [111] reciprocalspace vector $\mathbf{g}$ [20]. Analysis of the offset in the diffuse streaking as a function of $\mathrm{Nb}$ content in the $\mathrm{Zr}-\mathrm{Nb}$ system indicated that the magnitude of the offset decreased as the $\beta \rightarrow \omega$ transition was approached, with the diffuse streaking becoming sharp omega reflections inside the field of omega-stability [18].

Dark field electron microscopy performed right below the $\beta \rightarrow \omega$ transition temperature revealed the presence of $\langle 111\rangle$ rows of particles, each particle having a diameter of 10 to $15 \AA$ [18]. As we shall see in Section 3, such row morphology can be easily rationalized in terms of rather convincing thermodynamic arguments. Recently, by means of high resolution electron microscopy in $\mathrm{Zr}-\mathrm{Nb}$ alloys, Kuan and Sass [24] have been able to resolve what appears to be small omega-like particles in a concentration-temperature region outside the field of omega stability. The interpretation of such images is not straightforward, however, and the detailed displacement field associated with such particles still remains uncertain.

Some of the drawbacks of electron diffraction experiments, such as double diffraction and the inability of separating elastic and inelastic scattering, have been overcome by recent neutron diffraction and Mössbauer spectroscopy studies in $\mathrm{Zr}-\mathrm{Nb}$ alloys. The experimental evidence produced by neutron and Mössbauer diffuse scattering can be summarized as follows :

1) Analysis of the scattered intensity near an omega superlattice reflection as a function of the neutron energy transfer $\Delta E$ (see figure 2) reveals the presence of a strong peak at $\Delta E=0$, the so-called central peak [22]. The central peak represents elastically scattered intensity, resulting from static - or near static displacements.

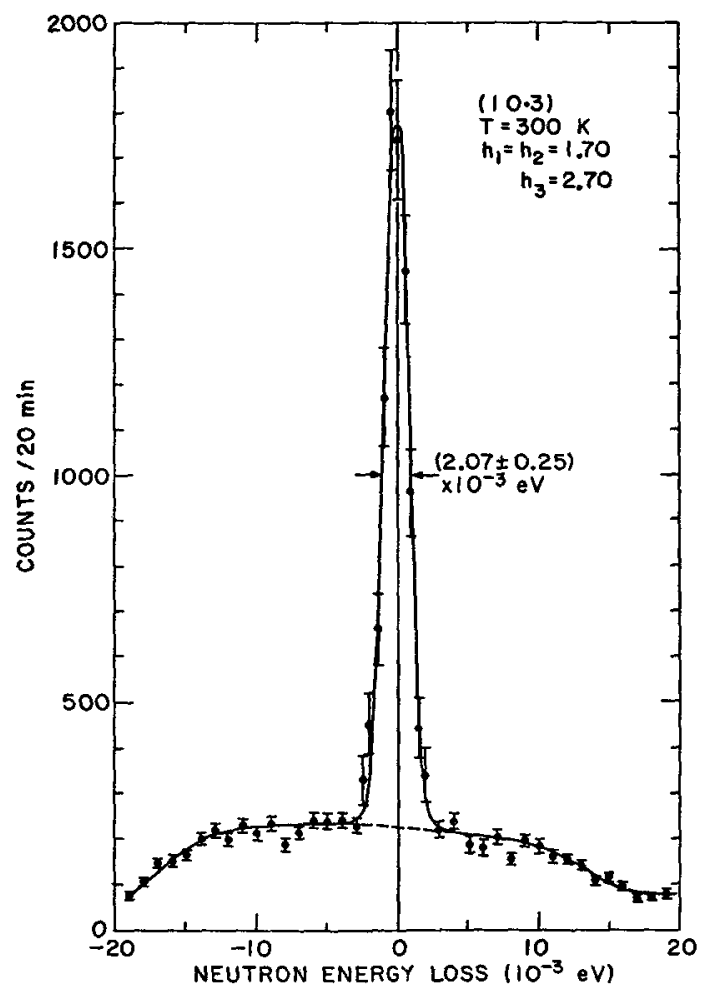

FIG. 2. - The central peak and overdamped phonon response as observed by neutron diffraction experiments above the $\beta \rightarrow \omega$ transition temperature [22].

2) The central peak persists over a very wide temperature range inside the $\beta$ phase, even up to $1000^{\circ} \mathrm{C}$ above the $\beta \rightarrow \omega$ transition in the case of $\mathrm{Zr}-20$ at. $\% \mathrm{Nb}[22]$.

3) The elastically scattered neutron intensity is offset from $\mathbf{k}_{\omega}$ in a manner consistent with earlier electron diffraction experiments. Furthermore, recent Mössbauer diffuse scattering studies appear to indicate that the magnitude of the offset increases as one moves further away from the origin of reciprocal space [23]. 
4) On a $(110)_{\beta}$ reciprocal lattice plane (figure 3), where superlattice reflections are given by

$$
\mathbf{k}_{\mathrm{s}}=\mathbf{k}_{\beta} \pm \mathbf{k}_{\omega}
$$

with $\mathbf{k}_{\beta}$ a bec reciprocal lattice vector and

$$
\mathbf{k}_{\omega}=1 / 3 \quad[\overline{11} 2],
$$

it is found that the diffuse intensity near $\mathbf{k}_{\beta}+\mathbf{k}_{\omega}$ is much weaker than that near $\mathbf{k}_{\beta}-\mathbf{k}_{\omega}$ for

$$
\mathbf{k}_{\beta} \cdot[111]>0,
$$

the opposite being true if the sign of $\mathbf{k}_{\beta}$ is reversed $[22,23]$.

5) The inelastically scattered intensity is characterized by the absence of well defined phonon peaks at finite values of the neutron energy transfer (see figure 2), in addition to a conspicuous lack of bcc symmetry [25].

As we shall see in the remainder of this paper, the experimental facts listed above have provided important clues in the development of phenomenological models for the $\beta \rightarrow \omega$ transition. Such theories have proved very valuable in explaining peculiar macroscopic phenomena associated with the group IV B transition metals, such as anomalies in internal friction [26] and diffusional behaviour $[12,27]$ close to the transition temperature.

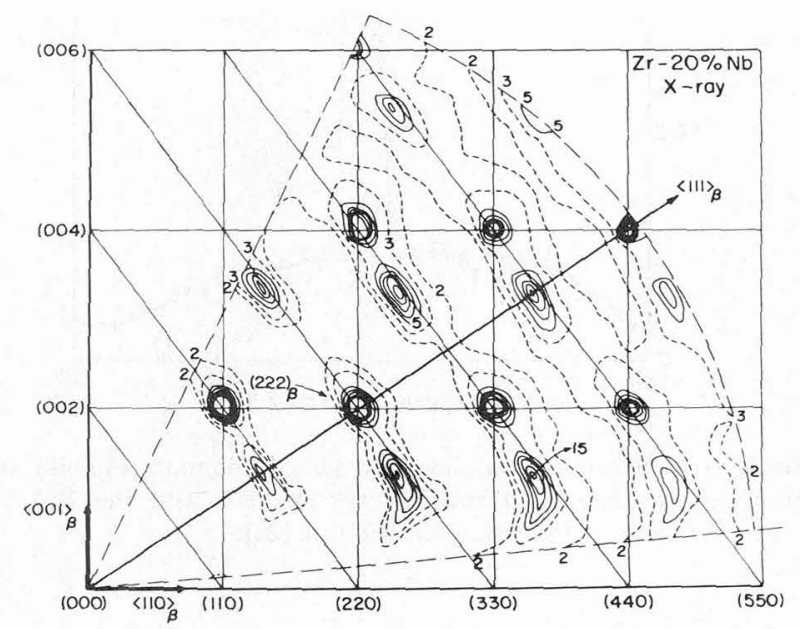

Fig. 3. - Diffuse intensity contour map on a $(110)_{\beta}$ section of reciprocal space [23].

2. The nature of the transformation. - Early studies of the $\beta \rightarrow \omega$ phase transformation in alloys of Group IV B transition metals emphasized the compositional aspects of the transformation, i.e., the partitioning of solute between the omega particles and the retained $\beta$ matrix. Only later, when it was recognized that the $\beta \rightarrow \omega$ transformation could take place at low temperatures, where solute mobility is extremely low, or under high pressure for the pure elements, it became apparent that the purely displacive nature of the transformation must provide the key to the transformation mechanism. Hence, in current theoretical treatments, lattices sites are assumed to be occupied by unspecified average atoms, of mixed $\mathrm{Zr}-\mathrm{Nb}$ character, for example. The natural order parameters are thus considered to be thermal averages of the atomic displacements from the bcc lattice of the high-temperature $(\beta)$ phase. Compositional effects should really be introduced, as displacive-compositional wave coupling may well be found to play an important role. In this brief review, however, only displacive effects will be considered.

The free energy of the system can then be written, in principle, as a Landau expansion in powers of atomic displacements [9-11]. In a simplified treatment, the amplitude of the $\mathbf{k}_{\omega}=2 / 3$ [111] wave is taken to be the unique order parameter $\eta$, and the Landau free energy expression can then be written as

$$
\Delta F=A \eta^{2}+B \eta^{3}+C \eta^{4}+\cdots
$$

where $\Delta F$ is the difference in free energy between the $\beta$ phase with and without displacements, $A, B$, and $C$ being temperature (and pressure) dependent phenomenological coefficients. A schematic plot of $\Delta F$ as a function of the order parameter $\eta$ is shown in figure 4 . For the case of a non-vanishing third-order free energy coefficient, the order parameter, determined by the minimization of the free energy, is zero at high temperatures and jumps discontinuously to a finite value $\eta_{0}$ at the transition temperature; thus the transition is first-order. This simplified picture does not strictly apply to the $\beta \rightarrow \omega$ transition where, as we shall see, a single homogeneous (independent of position in the crystal lattice) order parameter is not sufficient for a complete characterization of the transformation. Nevertheless, the presence of third-order anharmonicity in the atomic displacements is a convincing argument in favor of a first-order transition, although it should be pointed out that the order of the $\beta \rightarrow \omega$ transition has not yet been determined experimentally.

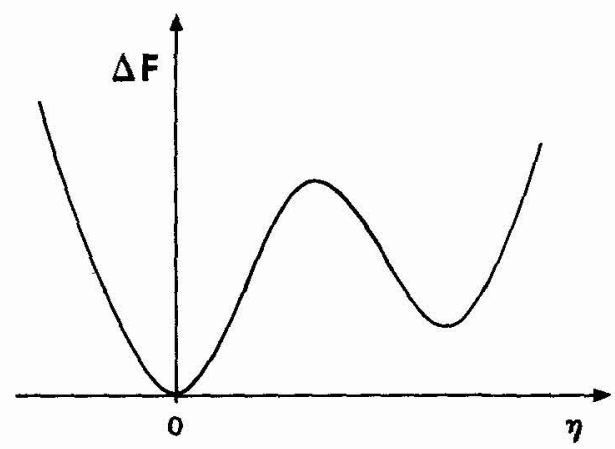

FIG. 4. - Characteristic shape of the free energy expansion for a first-order transition. 
It is interesting to note that most bec structures show a natural softening of the longitudinal phonon frequencies for a wave vector close to $2 / 3$ [111], the Brillouin zone center of the omega phase and a zone boundary point for the bcc phase. Such softening, shown in figure 5 as a dip in the longitudinal phonon frequencies at $\mathbf{k}_{\mathbf{m}} \simeq 0.71[111$, is structural in origin, arising from the unscreened Coulombic interactions of the ions with the uniform electron gas [28]. In the particular case of the Group IV B transition metals, additional softening of the short wavelength phonons may arise from screening effects associated with nesting or with flat portions of the Fermi-surface. In fact, recent calculations by Myron et al. [29] in

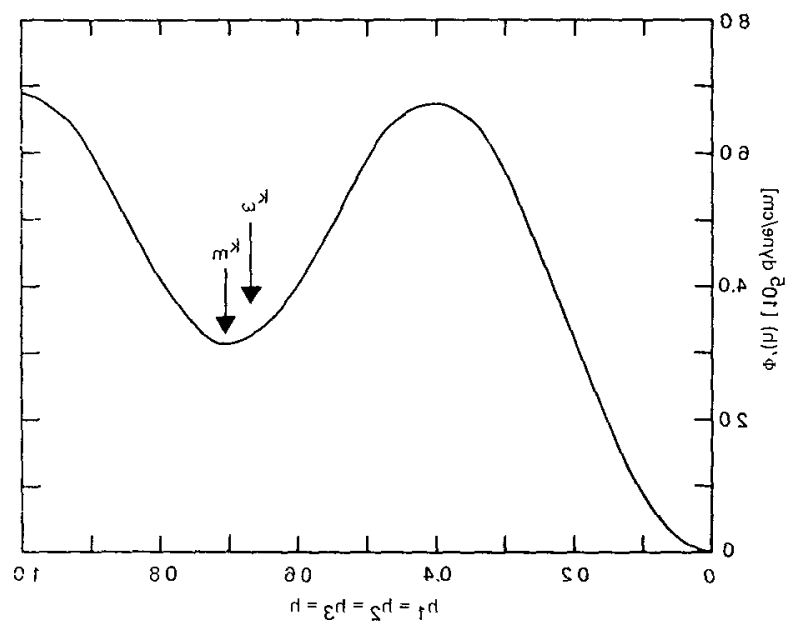

FIG. 5. - The energy modulus, proportional to the square of the harmonic phonon frequencies, for the [111] longitudinal branch, calculated by Cook [11].

$\mathrm{Zr}-\mathrm{Nb}$ alloys, show that the generalized susceptibility function has pronounced peaks near the $\mathbf{k}_{\omega}$ position in reciprocal space, an indication that the natural dip in the phonon frequencies (figure 5) will be considerably enhanced by electron screening. Thus the calculations by Myron et al. [29], appear to indicate that the $\beta \rightarrow \omega$ phase transformation is driven by strong electronphonon coupling, a likely mechanism being the formation of charge density waves (CDW). The effect of CDW's on a number of crystal properties has been studied by Overhauser [30] and by Chan and Heine [31]. In particular, the latter authors predict that a structural transition driven by CDWs will be of the classical soft mode type, i.e., one or more normal mode frequencies will vanish at the transition temperature. At the present time, the experimental evidence is not sufficient to determine whether or not the $\beta \rightarrow \omega$ phase transformation can be classified as a soft-mode transition. However, in view of the fact that a softmode mechanism is one of the simplest and better understood process giving rise to a structural transition, it is of some interest to review briefly the basic principles involved. Of necessity, we will consider a simplified model which, although not directly applicable to the $\beta \rightarrow \omega$ transition, will help to clarify the type of difficulties likely to be encountered in a more general treatment. A model soft mode transition can be treated quite independently of the microscopic softening mechanism by postulating that the crystal structure is unstable at low temperatures with respect to a given normal mode displacement, i.e., the harmonic frequency-squared associated with the normal mode in question is negative [32]. The simplest Hamiltonian which will show such a structural phase transformation is of the form

$$
\mathfrak{H}=\mathrm{H}_{2}+\mathrm{H}_{4}
$$

where $\mathrm{H}_{2}$, in terms of the frequencies $\omega(s, \mathbf{k})$ of the $s$-th mode of wave vector $\mathbf{k}$ and of the normal mode displacement aplitudes $Q(s, \mathbf{k})$ is given by

$$
H_{2}=\frac{1}{2} \sum_{\mathbf{k}, s} \omega^{2}(s, \mathbf{k}) Q(s, \mathbf{k}) Q(s,-\mathbf{k})
$$

and where $\mathrm{H}_{4}$ contains fourth-order products of the form $Q\left(s_{1}, \mathbf{k}_{1}\right) Q\left(s_{2}, \mathbf{k}_{2}\right) Q\left(s_{3}, \mathbf{k}_{3}\right) Q\left(s_{4}, \mathbf{k}_{4}\right)$, with $\mathbf{k}_{1}+\mathbf{k}_{2}+\mathbf{k}_{3}+\mathbf{k}_{4}$ equal to a reciprocal lattice vector of the high temperature structure. For the soft mode $\left(s, \mathbf{k}_{\mathrm{c}}\right)$, the square of the frequency $\omega\left(s, \mathbf{k}_{\mathbf{c}}\right)$ will be negative and the Hamiltonian as a function of the soft normal mode displacements will exhibit a characteristic double well potential. A new set of frequencies $\Omega(s, \mathbf{k})$ can be calculated in a straightforward manner by means of the Hartree or self-consistent phonon approximation [33]. The Hartree approximation consists in replacing in $\mathrm{H}_{4}$ products of the type $Q Q Q Q$ by $\langle Q Q\rangle Q Q$ where the brackets stand for themal averages calculated self-consistently in the harmonic approximation. The new set of temperature-dependent frequencies $\Omega(s, \mathbf{k})$ are then given in this approximation by

$$
\begin{aligned}
& \Omega^{2}(s, \mathbf{k})=\omega^{2}(s, \mathbf{k})+ \\
& \quad+k_{\mathrm{B}} T \sum_{s^{\prime} \mathbf{k}^{\prime}} \Phi\left(s, \mathbf{k} ; s^{\prime}, \mathbf{k}^{\prime}\right) / \Omega^{2}\left(s^{\prime}, \mathbf{k}^{\prime}\right)
\end{aligned}
$$

where $\Phi\left(s, \mathbf{k} ; s^{\prime}, \mathbf{k}^{\prime}\right)$ are effective fourth-order coupling constants and where it has been implicitly assumed that $k_{\mathrm{B}} T \gg \hbar \Omega$.

In the so-called quasiharmonic treatment [34], Eq. (2) is further approximated by replacing $\Omega^{2}(s, \mathbf{k})$ on the right hand side by the harmonic frequencies $\omega^{2}(s, \mathbf{k})$. In the quasiharmonic approximation, it can be shown that the order parameter, defined as the thermal average of the soft normal mode displacements $\left\langle Q\left(s, \mathbf{k}_{\mathrm{c}}\right)\right\rangle$, vanishes continuously when the transition temperature $T_{0}$ is approached from below, and consequently the transition is then second order [34]. Furthermore, on the high temperature side, the softmode frequency vanishes at $T_{0}$ as :

$$
\Omega^{2}\left(s, \mathbf{k}_{\mathrm{c}}\right)=A\left(T-T_{0}\right)
$$


with

$$
A=k_{\mathfrak{B}} \sum_{s, \mathbf{k}} \Phi\left(s, \mathbf{k}_{\mathrm{c}} ; s, \mathbf{k}\right) / \omega^{2}(s, \mathbf{k})
$$

where the transition temperature is given by

$$
T_{0}=-\omega^{2}\left(s, \mathbf{k}_{\mathrm{c}}\right) / A \text {. }
$$

Considering the fact that most displacive transitions are first-order, and the $\beta \rightarrow \omega$ transformation certainly appears to be so, the results produced by the quasiharmonic approximation are not particularly attractive. It turns out, however, that if Eq. (2) is solved by relaxing the quasi-harmonic approximation, the transformation becomes first order, with the softmode frequency vanishing at the instability temperature $T_{\mathrm{i}}\left(T_{\mathrm{i}}<T_{0}\right)$ as [35]

$$
\Omega^{2}\left(s, \mathbf{k}_{\mathfrak{c}}\right) \propto\left(T-T_{\mathrm{i}}\right)^{2} .
$$

The picture of the transition becomes now much more realistic since, in addition of predicting a first order transformation, the soft-mode frequencies do not vanish at $T_{0}$, in agreement with recent experimental evidence in the transition metal dichalcogenides [36].

The simple anharmonic perturbation method used for handling the Hamiltonian of Eq. (1) fails to reproduce one of the most conspicuous features observed in a number of structural transitions, namely the presence of a central peak in the dynamic phonon response (see figure 2). The central peak can, however, be formally recovered by means of some dissipative relaxation process coupled linearly to the normal displacive modes [37, 38]. This point has recently been emphasized, in connection with the transition metal dichalcogenides, by Bhatt and McMillan [38] who proposed a linear coupling between CDWs, of amplitudes $\breve{\zeta}(\mathbf{k})$, and the normal modes $Q(\mathbf{k})$, through a free energy of the form :

$$
\begin{aligned}
F=\sum_{\mathbf{k}}\{c(\mathbf{k}) \xi(\mathbf{k}) \xi(-\mathbf{k})+v(\mathbf{k})[\xi(\mathbf{k}) Q(-\mathbf{k})+ \\
+\xi(-\mathbf{k}) Q(\mathbf{k})]+\kappa(\mathbf{k}) Q(\mathbf{k}) Q(-\mathbf{k})\}
\end{aligned}
$$

where $\varepsilon(\mathbf{k}), v(\mathbf{k})$ and $\kappa(\mathbf{k})$ are phenomenological parameters.

In a rather different approach, the presence of the central peak is simply attributed to static or quasistatic displacive defects which persist well inside the high temperature phase $[39,40]$. Furthermore, the central peak may be a manifestation of soliton modes, as described briefly below. In summary, the softening associated with structural transitions can be understood quite well in terms of a simple anharmonic perturbation analysis. The transition is first-order when treated self-consistently even for the simple Hamiltonian given by Eq. (1), with the soft-mode frequency vanishing at the instability temperature. On the other hand, the central peak cannot be easily incorporated into the description and either static displacements or some dissipative relaxation process coupled to the displacive normal modes has to be invoked.

Although the description of structural phase transformations given above explains many of the features observed experimentally, the question remains whether or not a similar approach is applicable to the $\beta \rightarrow \omega$ transition. The main difficulty is that, in the $\beta \rightarrow \omega$ transition, third-order anharmonic coupling between normal modes play a significant role. These thirdorder anharmonicities represent a considerable mathematical complication since they cannot be properly treated in the simple Hartree or first-order selfconsistent approximation. Thus, higher levels of approximations are called for, from which one would expect to establish a clear connection between the static and dynamic aspects of the problem.

A particularly promising approach is that given recently by Varma [41] for the analytical solution of the equations of motion for an anharmonic linear chain. The solutions, differing markedly from those obtained by conventional perturbation methods, are found to involve soliton modes, which may be interpreted as moving domains. Of course, results valid for a linear chain, treated as a continuum, cannot be extrapolated directly to three-dimensional transformations in crystals. Nevertheless, certain conclusions appear to carry over to the $\beta \rightarrow \omega$ transformation which has marked one-dimensional character (along $\langle 111\rangle$ ). Certainly, the prediction concerning the existence of antiphase domains and resulting central phonon peak apply well to the omega case, although, because of the finite extent in three dimensions of omega and of smaller antiomega $(\omega)$ domains, with attendent threedimensional strain fields, one does not expect these domains to interpenetrate without mutually interacting, even if they belong to the same variant, as classical solitons do. Also solute-displacement coupling may considerably slow down the moving, and now interacting, domains.

3. Phenomenological models for the free energy. Cook has recently proposed a Landau expansion for the free energy in terms of the atomic displacements $[10,11]$. This approach has proved very helpful in understanding the static properties of the Group IV B transition metals such as the origin, offset and marked asymmetry in reciprocal space of the omega (central) peak. A key element of the model is the distinction between the $\omega$ and the $\bar{\omega}$ structures causing the free energy expansion to include strong third-order anharmonic contributions. Thus the free energy change due to static displacements will be given by

$$
\Delta F=F_{2}+F_{3}+F_{4}
$$

where $F_{2}, F_{3}$ and $F_{4}$ stand, respectively, for quadratic, cubic and quartic terms in the displacements, higherorder terms being neglected in order to keep the 
discussion simple. The second-order term, $F_{2}$, is the familiar one obtained from lattice dynamics in the harmonic approximation and it is given by

$$
F_{2}=\frac{1}{2} \sum_{\alpha \beta} \sum_{\mathbf{k}} \Phi_{\alpha \beta}(\mathbf{k}) U_{\alpha}(\mathbf{k}) U_{\beta}(\mathbf{k})
$$

in which $\alpha$ and $\beta$ refer to the Cartesian components of the displacements amplitudes $U(\mathbf{k})$. The free energy coefficients $\varphi_{\alpha \beta}(\mathbf{k})$ are expected to behave in a manner very similar to the harmonic phonon frequencies, showing marked softening at $\mathbf{k}_{\mathrm{m}} \simeq 0.71$ [111] (see figure 5). The anharmonic contributions to the free energy, $F_{3}$ and $F_{4}$, will be of the form

$$
\begin{array}{r}
F_{3}=\frac{1}{3 !} \sum_{\alpha \beta \gamma} \sum_{\mathbf{k} \mathbf{k}^{\mathbf{k}^{\prime \prime}}} \Phi_{\alpha \beta \gamma}\left(\mathbf{k}^{\prime}, \mathbf{k}^{\prime \prime}\right) U_{\alpha}(\mathbf{k}) U_{\beta}\left(\mathbf{k}^{\prime}\right) U_{\gamma}\left(\mathbf{k}^{\prime \prime}\right) \times \\
\times \delta\left(\mathbf{k}+\mathbf{k}^{\prime}+\mathbf{k}^{\prime \prime}, \mathbf{k}_{\beta}\right) \\
F_{4}=\frac{1}{4 !} \sum_{\alpha \beta \alpha^{\prime} \beta^{\prime} \mathbf{k k}_{\mathbf{k}} \mathbf{k}_{\mathbf{k}^{\prime \prime} \mathbf{k}^{\prime \prime \prime}} \Phi_{\alpha \beta \alpha^{\prime} \beta^{\prime}}\left(\mathbf{k}^{\prime}, \mathbf{k}^{\prime \prime}, \mathbf{k}^{\prime \prime \prime}\right) U_{\alpha}(\mathbf{k}) U_{\beta}\left(\mathbf{k}^{\prime}\right) \times} \\
\times U_{\alpha^{\prime}}\left(\mathbf{k}^{\prime \prime}\right) U_{\beta}\left(\mathbf{k}^{\prime \prime \prime}\right) \delta\left(\mathbf{k}+\mathbf{k}^{\prime}+\mathbf{k}^{\prime \prime}+\mathbf{k}^{\prime \prime \prime}, \mathbf{k}_{\beta}\right)
\end{array}
$$

where $\Phi\left(\mathbf{k}, \mathbf{k}^{\prime}\right)$ and $\Phi\left(\mathbf{k}, \mathbf{k}^{\prime}, \mathbf{k}^{\prime \prime}\right)$ are appropriate third and fourth-order free energy coefficients, respectively, and where the delta functions reflect the translational symmetry of the bcc lattice.

The offset in the elastic diffuse intensity can be understood by noting that the softening of the harmonic free energy coefficient $\Phi(\mathbf{k})$ is largest at a position $\mathbf{k}_{\mathrm{m}}$ in reciprocal space close to, but not exactly at the commensurate wave vector positon $\mathbf{k}_{\omega}$. Thus, an incommensurate displacive wave of wave vector $\mathbf{k}_{\mathrm{m}}$ will be favored by the harmonic free energy contribution. The structure developed by such an incommensurate wave will consist of a sequence of alternate $\omega$ and $\bar{\omega}$ like domains with both types of particles having the same volume fraction. It is apparent then that the free energy of the system can be lowered considerably if the volume fraction of $\bar{\omega}$ domains can be reduced by properly distorting the incommensurate plane wave. The distortion of the $\mathbf{k}_{\mathrm{m}}$ displacive wave will result in an increase in the harmonic free energy which can be more than compensated by the fact that the distorted wave can now reduce its energy by means of thirdorder anharmonic interactions. Note that an incommensurate plane wave cannot interact with itself through the third order anharmonic term, the only wave allowed to do so by the symmetry of the problem being the commensurate one $\mathbf{k}_{\omega}$.

A likely distortion for the incommensurate displacive wave is a phase modulation, the so-called phason, as recently shown by McMillan for a similar free energy expansion in terms of the amplitude of CDW's [42]. A detailed calculation of the phase modulation which minimizes the free energy is a rather complex problem and it will suffice here to emphasize that a properly distorted incommensurate wave can lower its free energy with respect to that of a single commensurate plane wave, as shown schematically in figure 6 . Thus, the fact that a distorted $\mathbf{k}_{\mathrm{m}}$ wave has a lower free energy provides a straightforward explanation for the observed offset in the diffuse intensity and, as shown elsewhere [43], for the marked $k$-space asymmetry in the intensity distribution (see figure 3).

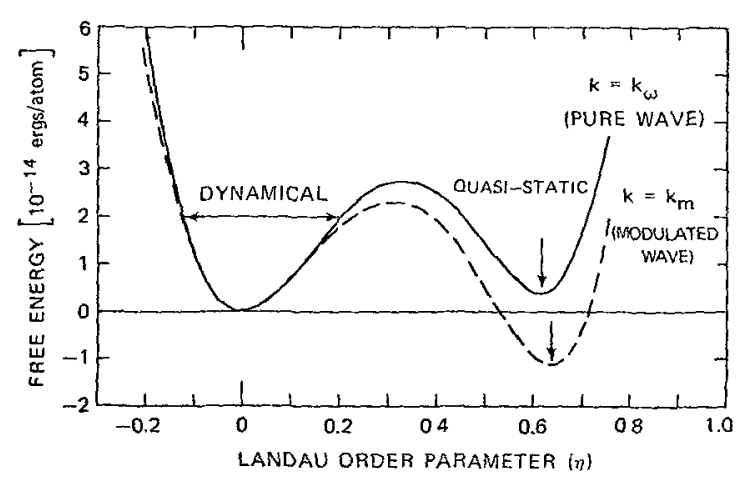

FiG. 6. - Expected behavior of the phenomenological free energy for a commensurate wave $\left(\mathbf{k}_{\omega}\right)$ and for a distorted $\mathbf{k}_{\mathrm{m}}$ wave (phason) [11].

In addition, the incommensurate wave approach appears to be in good agreement with the observation of $\langle 111\rangle$ rows of particles below the $\beta \rightarrow \omega$ transition temperature [18]. Such a microstructure has been explained by different models in terms of a $\mathbf{k}_{\mathrm{m}}$ displacive wave $[10,11,43]$ although some uncertainty remains concerning the exact displacement field of the particles.

Models for the free energy based on the concept of discrete displacements have also been proposed $[7,8,12]$. A common feature of such models is that the $\beta \rightarrow \omega$ transition is treated as an orderdisorder reaction where the ordering species are a discrete set of static atomic displacements. A particularly successful method for handling order-disorder phase transformations in general is the cluster variation method (CVM) proposed by Kikuchi [44]. This approach consists in a hierarchy of approximations in which the configurational entropy is written as a function of the distribution variables (concentrations) for different types of clusters of lattice points. A recent CVM calculation for the $\beta \rightarrow \omega$ transition, which uses the bcc primitive unit cell as the basic configurational cluster, has provided considerable insight into the problem of anomalous diffusion in the Group IV B transition metals [12]. Let us describe here the relevant aspects of such CVM calculation and in the next section, we shall discuss briefly the connection between anomalous diffusion and the $\beta \rightarrow \omega$ phase transformation.

In the CVM approach, the $\beta \rightarrow \omega$ phase transformation is modeled by associating to each bcc lattice point 
a displacement $u_{\mathrm{p}}$ along the $\langle 111\rangle$ direction of the form

$$
u_{\mathrm{p}}=u_{0} \sigma_{\mathrm{p}}
$$

where $u_{0}$ is half the distance between (111) planes and where the occupation operator $\sigma_{\mathrm{p}}$ takes values $0,+1$ or -1 . Proper ordering of the three types of displacements on different (111) planes of the $\alpha, \beta, \gamma$ bcc stacking sequence results in a particular subvariant of the omega phase.

The free energy of the system can be written as

$$
F=\sum_{\substack{i j k i \\ m n o p}} \varepsilon_{i j k l}^{m n o p} W_{i j k l}^{m n o p}-k_{\mathrm{B}} T \ln G_{\mathrm{N}}
$$

where $\varepsilon_{i j k i}^{m n o p}$ and $W_{i j k l}^{n n n p}$ are, respectively, the energies and concentrations of the 8-point clusters (bcc primitive unit cell) with the indices referring to the type of displacement $(0, \pm 1)$ at each corner of the unit cell. The combinatorial term $G_{\mathrm{N}}$ is the number of distinguishable configurations of the system having definite concentrations of the basic 8-point clusters, calculated in the cluster variation approximation [44].

At any temperature, the equilibrium distribution for the configurational variable $W$ can be obtained by minimizing the free energy given by Eq. (3). The minimization of the free energy is a rather cumbersome task since there are $3^{8}=6561$ cluster variables (not all distinct), and an equal number of simultaneous nonlinear algebraic equations to be solved. Such large numbers of simultaneous equations, are, nevertheless, tractable by relatively simple iteration techniques $[12$, 45]. The computational difficulties involved in the CVM calculations are, however, more than compensated by the fact that the temperature dependence of all intermediate cluster structures between the perfect bcc and perfect omega are obtained. Thus, a rather complete picture of the degree of short-range order in the system can be constructed.

As an example, figure 7 shows the temperature dependence of a particularly important cluster for diffusion, namely the activated complex consisting of a triangle of third-nearest neighbors collapsed onto the same plane with an atom at its center (see figure 8).

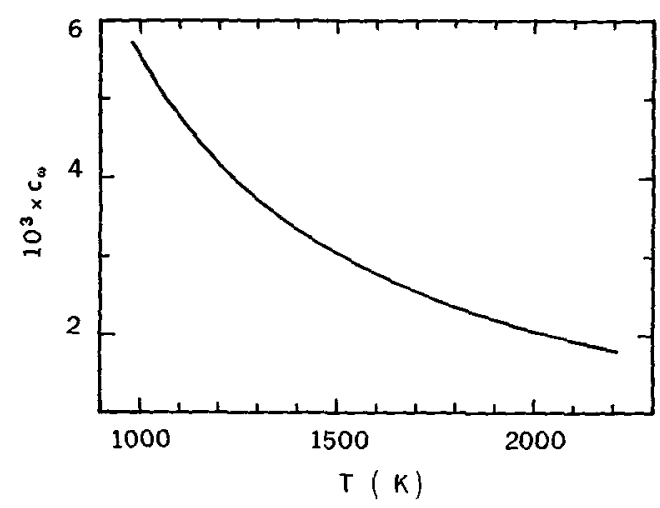

FIG. 7. - The concentration of $\bar{\omega}$ embryos (activated complexes for diffusion) versus the absolute temperature [12].

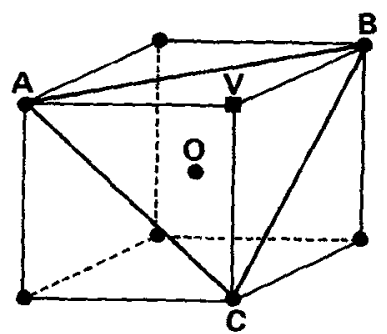

(a)

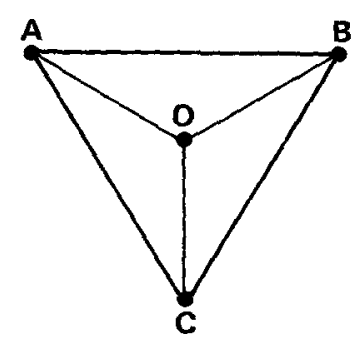

(b)

Frg. 8. - The configurations of the elementary $\omega$ embryo and the activated complex for diffusion. The atom at 0 exchange places with a vacancy at $\mathrm{V}(a)$ by forming, in the process, an elementary $\omega$ embryo $(b)$.

Such a cluster has the characteristic trigonal bonding of the omega structure and it will be termed $\omega$-embryo. Thus, it is not surprising to see in figure 7 that the concentration of omega-embryos increases considerably as the $\beta \rightarrow \omega$ transition temperature is approached which for $\mathrm{Zr}$ should be around $900 \mathrm{~K}$.

The treatment of displacive phase transformations by means of the CVM cannot be considered fully developed, however. Due to the displacive nature of the transformation, vibrational entropy changes will, in general, play an important role and the configurational free energy may not suffice for a complete characterization of the phase transition. Assuming, however, that such vibrational contributions can be properly taken into account, considerably larger clusters, and therefore more configurational variables than are used in the case of replacive transitions, appear to be necessary. The necessity of larger clusters arises from the relatively extended elastic fields associated with a given displacive defect. Thus, the practical application of the CVM to displacive transitions at the present time will require further improvements in the analytical techniques used for the minimization of the free energy.

4. Anomalous diffusion. - It is well established that the diffusion coefficient in the Group IV B transition metals $\mathrm{Ti}, \mathrm{Zr}$ and $\mathrm{Hf}$ deviates considerably from the normal Arrhenius relation

$$
D=D_{0} \exp (-Q / R T)
$$

where the pre-exponential factor $D_{0}$ and activation energy $Q$ are temperature-independent [46]. The ano- 
malous diffusional behavior in the Group IV B transition metals is characterized by a large enhancement in the diffusion coefficient at low temperatures, resulting in a markedly curved Arrhenius plot. Fitting the experimental data by means of the activated laws of the form

$$
D=D_{0} \exp (-Q / R T)+D_{0}^{\prime} \exp \left(-Q^{\prime} / R T\right)
$$

results in normal values of $D_{0}$ and $Q$ at high temperatures, i.e., $D_{0}$ falling between 0.05 and $5 \mathrm{~cm}^{2} / \mathrm{s}$ and $Q$ approximately given by

$$
Q=34 T_{\mathrm{m}}
$$

with $T_{\mathrm{m}}$ the melting temperature. At low temperature, however, the values of both $D_{0}^{\prime}$ and $Q^{\prime}$ are much too low to be rationalized in terms of any reasonable normal diffusion mechanism [46]. A particularly puzzling effect is observed in the diffusional behavior of $\beta$-stabilized alloys of $\mathrm{Ti}, \mathrm{Zr}$ and $\mathrm{Hf}:$ it is seen that, by increasing the solute content, the apparent activation energy for diffusion increases even though the melting (solidus) temperature $T_{\mathrm{m}}$ in most cases decreases [47]. In view of the empirical relation (4), such behavior defies interpretation in terms of conventional diffusion mechanisms.

Recently, a model for anomalous diffusion, closely connected to the $\beta \rightarrow \omega$ transition, has clarified the peculiar diffusional properties of the Group IV B transition metals [27]. The key element of the model is the identity of structures of the activated complex for diffusion and that of an omega-embryo, that is, the smallest unit or cluster of neighboring atoms having the essential omega-structure (see figure 8). Thus, in the case of systems for which the omega structure is a low temperature metastable phase, close to the transition temperature the free energy of formation of an activated complex (omega-embryo) will be much smaller than in the case of normal bcc metals, resulting in a higher concentration of activated complexes and consequently enhancing diffusion at low temperatures. On the other hand, in the limit of high temperatures, random thermal vibrations will tend to destroy the omega embryo, and the diffusion process will be characterized by normal values and behavior of the diffusion coefficient.

The increase in concentration $c_{\omega}$ of the omegaembryos (activated complexes) close to the $\beta \rightarrow \omega$ transition temperature is apparent from figure 7, where $c_{\omega}$ was calculated in the CVM approximation referred to in the last section [12].

The diffusion coefficient can now be calculated as

$$
D=\gamma a^{2} v_{0} c_{v} c^{*}
$$

where $\gamma$ is a numerical constant close to unity, $a$ is the lattice parameter, $v_{0}$ is the attempt frequency, $c_{\mathrm{v}}$ is the vacancy concentration and $c^{*}$ is the total concentration of activated complexes given by

$$
c^{*}=c_{n}+c_{\omega}
$$

with $c_{\mathrm{n}}$ the concentration of normal activated complexes. After some straightforward algebra, Eq. (5) can be written as

$D=D_{0} \exp (-Q / R T)\left[1+c_{\omega} \exp \left(\Delta G_{\mathrm{m}} / R T\right)\right]$

where $D_{0}$ and $Q$ are normal pre-exponential factor and activation energy, respectively, and where $\Delta G_{\mathrm{m}}$ is given by

$$
\Delta G_{\mathrm{m}}=\Delta H_{\mathrm{m}}-T \Delta S_{\mathrm{m}}
$$

with $\Delta H_{\mathrm{m}}$ and $\Delta S_{\mathrm{m}}$ the enthalpy and entropy of formation of normal activated complexes. When the concentration of omega embryos $c_{\omega}$ shown in figure (7) is used, Eq. (6) results in an excellent fit to the selfdiffusion data on $\mathrm{Zr}$ [48] with $D_{0}=1.3 \mathrm{~cm}^{2} / \mathrm{s}$, $Q=65.0 \mathrm{kcal} / \mathrm{mole}, \Delta H_{\mathrm{m}}=33.5 \mathrm{kcal} / \mathrm{mole}$, and $\Delta S_{\mathrm{m}}=2.3 \mathrm{R}$ (see figure 9). The agreement between the model and the experimental data shown in figure (9) is particularly relevant since all classical diffusion parameters used fall well within the range prescribed by the well-established semi-empirical rules of normal diffusion [46].

The increase in the apparent activation energy with the $\beta$-stabilizing alloying content can be explained equally well by the model, as recently shown in the câse of Ti-Mn alloys [12]. Actually, the $\beta \rightarrow \omega$ transition temperature decreases much more rapidly with Mn content than does the solidus temperature $T_{\mathrm{m}}$. Thus, at a given temperature, increased alloying effectively takes the system further away from the omega transition so that the concentration of omega embryos is expected to decrease, resulting in an increasingly normal behavior, as is observed [49].

Acknowledgments. - The authors are indebted to Dr. Ryoichi Kikuchi for many illuminating discussions concerning The Cluster Variation Method. This work was supported in part by The U.S. Army Research Office (Durham).

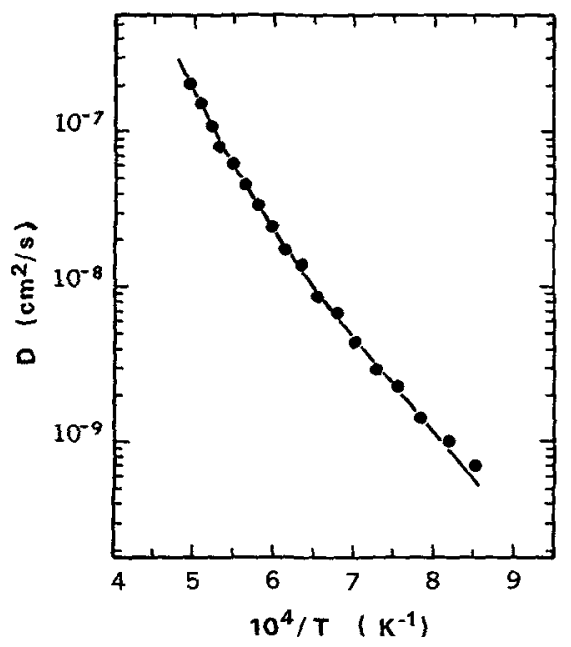

Fig. 9. - The fit of the self-diffusion coefficient calculated by the CVM to the experimental data on $\mathrm{Zr}$ obtained by Federer and Lundy [12, 48]. 


\section{References}

[1] Hickman, B. S.,.J. Mater. Sci. 4 (1969) 554.

[2] SAss, S. L., J. Less Common Metals 28 (1972) 157.

[3] Jamieson, J. C., Science 140 (1963) 72.

[4] Tittmann, B., Hamilton, D. and Jayaraman, A., J. Appl. Phys. 35 (1964) 732.

[5] Frost, P. D., Parris, W. N., Hirsch, L. L., Dorg, T. R. and SCHWARTZ, C. M., Trans. ASM 46 (1954) 231.

[6] Frost, P. D., Parris, W. N., Hirsch, L. L., Doig, T. R. and SCHwartz, C. M., Trans. ASM 46 (1954) 1056.

[7] De Fontanne, D. and Buck, O., Phil. Mag. 27 (1973) 967.

[8] De Fontaine, D. and Kikuchi, R., Acta Met. 22 (1974) 1139.

[9] Cook, H. E., Acta Met. 21 (1973) 239.

[10] Cook, H. E., Acta Met. 22 (1974) 1445.

[11] CooK, H. E., Acta Met. 23 (1975) 1041.

[12] SANChez, J. M., Ph. D. Dissertation, University of California, Los Angeles (1977).

[13] Bagaryatskiy, Yu. A., Nosova, G. I. and Tagunova, T. V., Dokl. Skod. Nouk SSSR 105 (1955) 1225.

[14] SilcoCk, J. M., Davies, M. H. and HARdy, H. K., The Mechanism of Phase Transformations in Solids (Inst. of Metals, London) 1956.

[15] Silcock, J. M., Acta Meta. 6 (1958) 48.

[16] Bagaryatskiy, Yu. A. and Nosova, G. I., Sov. Phys. Crystallogr. 3 (1958) 15.

[17] De Fontaine, D., Acta Met. 18 (1970) 275.

[19] De Fontaine, D., Paton, N. E. and Williams, J. C., Acta Met. 19 (1971) 1153.

[20] Paton, N. E., de Fontaine, D. and Williams, J. C., 29th Dun. Proc. Electron Microscopy Soc. Amer., Boston, Mass. (1971).

[21] Jackson, W. A., Perkins, A. J. and Hehemann, F., Met. Trans. 1 (1970) 2014.

[22] Moss, S. C., Keatrng, D. T. and Axe, J. D., Phase Transition, 1973, L. E. Cross, ed. (Pergamon Press, New York) 1973.

[23] Lin, W., Spalt, H. and Battermann, B. W., Phys, Rev. B 13 (1976) 5158.

[24] Kuan, T. S. and Sass, S. L., Report No. 2803, edited by The Materials Science Center, Cornell University, Ithaca, New York (1977).
[25] Axe, J. D., Keating, D. T. and Moss, S. C., Phys. Rev. Lett. 35 (1975) 530.

[26] Cook. H. E., Acta Met. (to be published).

[27] Sanchez, J. M. and De Fontaine, D., Phys. Rev. Lett. 35 (1975) 227.

[28] Price, D. L., Singwi, K. S. and Tosi, M. P., Phys. Rev. B 2 (1970) 2983.

[29] Mrron, H. W., Freeman, A. J. and Moss, S. C., Phys. Rev. Lett. (to be published).

[30] Overhauser, A. W., Phys. Rev. B 3 (1971) 3173.

[31] Chan, S. K. and Heine, V., J. Phys. F 3 (1973) 795.

[32] Silverman, B. D. and Joseph, R. I., Phys. Rev. 129 (1963) 2062.

[33] See from example WallaCE, D. C., Thermodynamic of Crystals (John Wiley and Sons, Inc., New York) 1972.

[34] Kwok, P. C. and Miller, P. B., Phys. Rev. 151 (1966) 387.

[35] Pytre, E., Phys. Rev. Lett. 28 (1972) 895.

[36] Moncton, D. E., AXe, J. D. and Di Salvo, F. J., Phys. Rev. Lett. 34 (1975) 734.

[37] Shirane, G. and Axe, J. D., Phys. Rev. Lett. 27 (1971) 1803.

[38] Bhatt, R. N. and McMillan, W. L., Phys. Rev. B 12 (1975) 2042.

[39] Varma, C. M., Phillips, J. C. and Chut, S. T., Phys. Rev. Lett. 33 (1974) 1223.

[40] Cook, H. E., Phys. Rev. B 15 (1977) 1477.

[41] Varma, M. C., Phys. Rev. B 14 (1976) 244.

[42] McMillan, W. L., Phys. Rev. B. 14 (1976) 1496.

[43] Sanchez, J. M. and De Fontaine, D., J. Appl. Cryst. 10 (1977) 442

[44] KiKUCH, R., Phys. Rev. 81 (1951) 988.

[45] KixuchI, R., J. Chem. Phys. 60 (1974) 1071.

[46] Le Claire, A. D., Diffusion in Body-Centered Cubic Metals (ASM, Metals Park, Ohio) 1965.

[47] Lazarus, D., Diffusion in Metals, Vol. 10, F. Seitz and D. Turnbull, ed. (Academic Press, New York) 1960.

[48] FEDERER, J. I. and Lundy, T. S., Trans. AIME 227 (1963) 592.

[49] Santos, E. and Dyment, F., Phil. Mag. 31 (1975) 809. 